\title{
Satisfação dos pacientes com os serviços de saúde mental: a percepção de mudanças como preditora
}

\author{
Patients' satisfaction with mental health services: \\ the perception of changes as predictor \\ Mônia Aparecida da Silva', Marina Bandeira², João Domingos Scalon³, Maria Amélia Cesari Quaglia
}

\section{RESUMO}

Objetivos: O principal objetivo deste estudo foi investigar as variáveis preditoras da satisfação dos pacientes com os serviços de saúde mental. Como objetivo secundário, avaliaram-se os níveis de mudança percebida e de satisfação dos pacientes. Método: Foi realizado um estudo de corte transversal, do tipo correlacional, com 110 pacientes psiquiátricos atendidos em três serviços públicos de saúde mental. Os pacientes foram entrevistados para aplicação da Escala de Mudança Percebida (EMP) e da Escala de Satisfação dos Pacientes com os Serviços de Saúde Mental (SATIS-BR). Resultados: O principal resultado encontrado foi que a percepção de mudança pelos pacientes constituiu a variável preditora mais importante da satisfação e, em segundo lugar, a maior idade. Foi detectada alta satisfação dos pacientes com os serviços e escores de percepção de mudança diferenciados em relação aos diferentes aspectos dos serviços avaliados. Conclusão: Este estudo revela a importância da percepção dos próprios pacientes sobre os resultados do tratamento como preditora da satisfação com os serviços. Os construtos de satisfação e percepção de mudança se revelaram importantes na avaliação dos serviços de saúde mental. Estudos longitudinais e com amostras maiores e aleatórias poderão fornecer dados adicionais para a reavaliação desses resultados.

\section{ABSTRACT}

Objectives: The main objective of this study was to evaluate the predicting factors of patients' satisfaction with mental health services. The secondary objective was to evaluate patients' levels of perceived changes and satisfaction. Method: A cross-sectional correlational study was carried out with 110 patients attending three public mental health services. Patients were interviewed for the application of the Perceived Changes Scale (EMP) and the Patient's Satisfaction with Mental Health Services Scale (SATIS-BR). Results: The main result was that patients' perceived change was the most important predictor of satisfaction with services and, in the second place, the greater age. It was found high levels of patients' satisfaction with mental health services and distinct levels of perceived change. Conclusion:

1 Universidade Federal de São João del Rei (UFSJ), Programa de Mestrado em Psicologia; UFSJ, Laboratório de Pesquisa em Saúde Mental (LAPSAM).

2 UFSJ; CNPq; Université de Montreal; McGill University; Université de Montréal.

3 Universidade Federal de Lavras (UFLA), Departamento de Ciências Exatas; Harvard University.

4 UFSJ, Departamento das Psicologias; Universidade de São Paulo (USP), Faculdade de Filosofia, Ciências e Letras de Ribeirão Preto (FFCLRP).

Instituição na qual foi realizada a pesquisa: Laboratório de Pesquisa em Saúde Mental (LAPSAM) da Universidade Federal de São João del Rei (UFSJ).

Endereço para correspondência: Mônia Aparecida da Silva

Departamento de Psicologia, Laboratório de Pesquisa em Saúde Mental (LAPSAM), UFSJ

Praça Dom Helvécio, 74

36301-160 - São João del Rei, MG

E-mail:moniapsi@yahoo.com.br 


\section{Keywords}

Patient satisfaction, mental

health services, outcome

assessment (health care).
This study points to the importance of patients' own perception about treatment outcome as predictor of satisfaction with mental health services. Satisfaction and perceived changes proved to be important in mental health evaluating. Longitudinal, random sampling and greater size sample studies may provide additional data for these results reevaluation.

\section{INTRODUÇÃO}

Com a desinstitucionalização psiquiátrica e a consequente reestruturação dos serviços de assistência em saúde mental, tem sido cada vez mais enfatizada a necessidade de avaliação dos serviços ${ }^{1}$. Diante da complexidade que envolve os sistemas de saúde e saúde mental, a avaliação é considerada como essencial para a garantia de qualidade dos serviços ${ }^{2,3}$.

A Organização Mundial de Saúde (OMS) destacou a necessidade de incorporar a avaliação dos resultados do tratamento como prática contínua e permanente, utilizando os dados da avaliação para melhorar a qualidade da assistência prestada ${ }^{3,4}$. De acordo com Hunter e Cameron ${ }^{5}$, a avaliação deve ser um procedimento de rotina dos serviços, podendo ser realizada pelos próprios profissionais da instituição. A abordagem de avaliação dos resultados dos serviços, recomendada pela OMS, é integrativa, ou seja, inclui a perspectiva dos pacientes, familiares e profissionais de saúde mental ${ }^{3}$.

A avaliação dos serviços, na perspectiva dos pacientes, tem sido destacada na literatura ${ }^{6,7}$. Os pacientes fornecem uma perspectiva única sobre o seu estado clínico e sobre os efeitos das intervenções em suas vidas ${ }^{6}$. Suas experiências com o tratamento e muitos dos resultados obtidos só podem ser experimentados e relatados por eles próprios, sendo inacessíveis a avaliações de terceiros ${ }^{8}$. A perspectiva dos pacientes, entretanto, tem sido vista com ressalvas por alguns clínicos e pesquisadores, que questionam a credibilidade dessas avaliações, por causa da dificuldade de insight de pacientes com transtornos graves e persistentes. Entretanto, pesquisas têm comprovado a confiabilidade de relatos de pacientes psiquiátricos estabilizados 9,10 , o que justifica a inclusão de sua perspectiva na avaliação dos resultados do tratamento.

Dentre os estudos de avaliação dos resultados, na perspectiva dos pacientes, a medida de satisfação com os serviços tem sido a mais frequentemente utilizada7,9,10. A satisfação dos pacientes está relacionada a diversos fatores, tais como: adesão ao tratamento, características do atendimento, variáveis sociodemográficas e clínicas dos pacientes, tipos de intervenção, redução da sintomatologia, competência da equipe, qualidade da relação entre terapeuta e cliente, acessibilidade aos serviços, qualidade das instalações, continuidade dos cuidados e também a informação recebida ${ }^{11,12}$.

Recentemente, outra medida de resultados na perspectiva dos pacientes tem se destacado na avaliação dos serviços. Trata-se da percepção de mudanças pelos pacientes em função do tratamento recebido. A medida de percepção de mudanças se refere aos efeitos do tratamento na saúde física e psicológica, na vida social e nas atividades, tal como percebido pelo próprio paciente. Alguns autores apontaram a importância dessa medida na avaliação dos resultados do tratamento ${ }^{6,7}$. Pesquisas recentes encontraram relações significativas entre a percepção de mudança pelos pacientes e as variáveis referentes à alteração na sintomatologia psiquiátrica ${ }^{6,13-16}$, qualidade de vida ${ }^{17}$ e percepção de mudança pelos familiares ${ }^{18}$. No entanto, a relação específica entre a percepção de mudança e a satisfação dos pacientes com os serviços tem sido pouco investigada.

No contexto internacional, foram encontrados, nas bases de indexadores Bireme, Medline, Lilacs, Sibinet-USP e PsycINFO, apenas cinco estudos que investigaram a relação entre mudanças percebidas pelos pacientes como resultado do tratamento e sua satisfação com os serviços de saúde mental 7,16,19-21. Todos esses estudos verificaram a existência de uma relação positiva significativa entre mudanças terapêuticas percebidas pelos pacientes e sua satisfação com os serviços.

Porém, esses estudos apresentam algumas limitações. Quatro deles ${ }^{16,19-21}$ utilizaram instrumentos dicotômicos e unifatoriais, que são considerados pouco adequados para avaliar essas variáveis. Escalas de medidas dicotômicas possuem baixa sensibilidade, pois não oferecem diversidade de alternativas de respostas. Escalas unifatoriais não fornecem escores diversificados para aferir as diferentes dimensões do construto avaliado, permitindo apenas uma avaliação global da variável estudada22. Escalas multifatoriais, ao contrário, possuem subescalas diferenciadas que avaliam diversas dimensões do construto, por meio de escores independentes. Essas limitações, que podem interferir na confiabilidade dos dados, apontam para a necessidade de se realizarem outros estudos, nessa temática, que incluam a utilização de instrumentos multidimensionais.

No contexto brasileiro, até o momento, não foi encontrada nenhuma pesquisa, nas bases de dados do SciELO, Sibinet-USP, Lilacs e Medline, que tenha estudado a relação entre percepção de mudanças e satisfação dos pacientes psiquiátricos com os serviços. Foram encontrados apenas estudos que avaliaram, isoladamente, uma dessas variáveis, sem relacioná-las. Cinco estudos avaliaram quantitativamente a satisfação dos pacientes com os serviços ${ }^{23-27}$ e três avaliaram a percepção de mudanças pelos pacientes ${ }^{14,17,18}$. 
Dentre esses três últimos estudos, um deles avaliou a relação entre percepção de mudança pelos pacientes e a alteração da sintomatologia ${ }^{14}$, o outro investigou a relação entre percepção de mudança e qualidade de vida ${ }^{17}$ e um terceiro comparou a percepção de mudança de pacientes e de seus familiares ${ }^{18}$. Tendo em vista a carência de investigações que avaliem a relação entre essas duas variáveis, o principal objetivo desta pesquisa foi avaliar se a percepção de mudança pelos pacientes em função do tratamento recebido é uma variável preditora mais importante de sua satisfação com os serviços do que outras variáveis sociodemográficas e clínicas.

\section{MÉTODO}

\section{Delineamento do estudo}

Trata-se de um estudo do tipo correlacional e de corte transversal ${ }^{28,29}$, no qual as medidas de satisfação e de percepção de mudança foram tomadas em um único intervalo de tempo. Consiste também de uma pesquisa avaliativa, do tipo somativa, definida, na classificação de Selltiz et al. ${ }^{28}$ e de Contandriopoulos et al. ${ }^{29}$, como aquela pesquisa que visa avaliar os resultados ou efeitos de serviços ou programas de tratamento.

\section{Participantes}

Participaram deste estudo 110 pacientes psiquiátricos que apresentavam sintomatologia estável no período da coleta de dados e que recebiam tratamento, do tipo ambulatorial, em três instituições públicas de saúde mental. A amostra foi do tipo não probabilístico e os participantes foram selecionados por meio de seus prontuários e das indicações dos profissionais dos serviços. Foram incluídos pacientes adultos, maiores de 18 anos, que faziam tratamento há, pelo menos, um ano. Todos os pacientes recebiam tratamento psiquiátrico medicamentoso e apenas 4,50\% da amostra recebiam também tratamento psicológico.

\section{Instrumentos de medida}

Avaliação da satisfação: A satisfação dos pacientes com os serviços foi avaliada por meio da versão abreviada da Escala de Avaliação da Satisfação dos Pacientes com os Serviços de Saúde Mental (SATIS-BR). Essa escala foi elaborada pela divisão de Saúde Mental da Organização Mundial de Saúde ${ }^{30}$ e validada para o Brasil por Bandeira et al. ${ }^{31}$. Trata-se de uma escala multidimensional, possuindo duas subescalas: 1. Satisfação com os Profissionais e 2. Satisfação com o Serviço. A SATIS-BR possuiu 13 itens, sendo nove deles agrupados nas duas subescalas e quatro itens adicionais incluídos na escala global. As alternativas de resposta aos itens estão dispostas em uma escala do tipo Likert com 5 pontos, na qual 1 representa que o usuário está muito insatisfeito e 5 que ele está muito satisfeito. A escala SATIS-BR apresentou propriedades psicométricas adequadas de fidedignidade, avaliada pela sua consistência interna, de validade de construto e de validade concomitante com a escala Client Satisfaction Questionnaire (CSQ-8) ${ }^{31}$.

Avaliação da percepção de mudança: A Escala de Mudança Percebida (EMP) foi elaborada por Mercier et al. ${ }^{6}$, no Canadá, e validada para o Brasil por Bandeira et al..$^{32}$. Essa escala avalia os resultados do tratamento na perspectiva dos próprios pacientes, ou seja, o impacto real das intervenções em suas vidas, conforme percebido por eles. Trata-se de uma escala multidimensional, contendo três subescalas: 1. Ocupação e Saúde Física; 2. Dimensão Psicológica e Sono; e 3. Relacionamentos e Estabilidade Emocional. A escala possui 19 itens, estando 18 deles dispostos nas três subescalas e um item é de avaliação global do tratamento. As alternativas de resposta a cada item estão dispostas em uma escala do tipo Likert de 3 pontos, indicando que um dado aspecto da vida do paciente está: 1. pior do que antes, 2. sem mudança e 3. melhor do que antes. A escala EMP apresenta propriedades psicométricas adequadas de fidedignidade, avaliada pela consistência interna e estabilidade temporal, e de validade de construto, confirmada por análise fatorial ${ }^{32}$.

\section{Coleta de dados}

Os participantes foram entrevistados pela primeira autora e por quatro estagiários de Psicologia, previamente treinados. O treinamento incluiu preparação teórica e realização de entrevistas supervisionadas por pesquisadores, com experiência na aplicação das escalas. A coleta de dados foi feita, por meio de entrevistas estruturadas, nos serviços de saúde mental que participaram da pesquisa ou nas residências dos sujeitos, após agendamento prévio e conforme sua disponibilidade. Foram aplicadas as escalas EMP e SATIS-BR, com duração aproximada de 50 minutos. A compreensão dos pacientes acerca das questões das escalas foi verificada utilizando-se a Técnica de Sondagem, denominada originalmente como Probe Technique ${ }^{33}$. Essa técnica consiste em solicitar aos pacientes que justifiquem suas respostas a cada um dos itens das escalas. As variáveis sociodemográficas e clínicas dos pacientes foram coletadas por meio de um questionário, construído de acordo com a literatura e testado em estudos anteriores.

\section{Análise de dados}

Os dados foram computados e analisados por meio do programa SPSS, versão 13.0. As análises estatísticas foram propostas com base nas obras de Hosmer e Lemeshow ${ }^{34}$, Dancey e Reidy ${ }^{35}$ e Marques de Sá ${ }^{36}$. Foram utilizados testes paramétricos para as análises estatísticas, após ter sido constatada a normalidade dos dados para as duas escalas, por meio do teste de Kolmogorov-Smirnov (EMP: K-S = 1,31; $p=$ $0,07$ e SATIS-BR: $\mathrm{K}-\mathrm{S}=1,31 ; p=0,60)$. 
Foi utilizada a análise estatística descritiva, com cálculo de médias, desvios-padrão e porcentagens para avaliar as características sociodemográficas e clínicas da amostra, os escores de satisfação e os níveis de percepção de mudança. As médias dos itens de cada escala foram comparadas pela análise de variância ANOVA e pelo teste post hoc de Tukey, para verificar se havia diferenças significativas entre elas.

Foi feita uma análise multivariada para identificar as variáveis preditoras da maior satisfação dos pacientes com os serviços, tendo como variável dependente o escore global de satisfação e, como variáveis independentes, as variáveis sociodemográficas e clínicas dos pacientes e a percepção de mudança. Para selecionar as variáveis sociodemográficas e clínicas que entrariam no modelo de regressão, foi feita primeiramente uma análise univariada utilizando o teste $t$ de Student e a correlação de Pearson ${ }^{36}$. Foram incluídas as variáveis que se mostraram significativas nas análises univariadas $(p<0,05)$, assim como as variáveis que atingiram o valor de $p \leq 0,25$. Segundo Hosmer e Lemeshow ${ }^{34}$, variáveis com $p \leq 0,25$ identificadas nas análises univariadas devem ser incluídas no modelo de regressão linear, pois elas podem ser significativas nas análises multivariadas. As variáveis sociodemográficas e clínicas significativas pré-selecionadas foram: idade, categoria diagnóstica, escolaridade, independência para tomar a medicação, local em que o paciente fazia tratamento psiquiátrico e número de crises no último ano. As variáveis com valor de $p \leq 0,25$ foram: comorbidade psiquiátrica, duração do transtorno psiquiátrico, duração do tratamento psiquiátrico, presença de doenças físicas, tipo de medicação e indicação de que o paciente tinha ou não renda.

A análise multivariada constou de duas regressões lineares múltiplas. A primeira incluiu as variáveis sociodemográficas e clínicas pré-selecionadas nas análises univariadas e o escore global de percepção de mudanças, para verificar a importância relativa da percepção de mudanças como variável preditora da satisfação com os serviços. A segunda análise de regressão incluiu somente os escores das três subescalas da EMP, visando identificar quais dimensões da percepção de mudança seriam mais preditoras da satisfação.

Os modelos de regressão foram construídos por meio da técnica stepwise. Em acordo com Montgomery e Peck ${ }^{37}$, e considerando que a SATIS-BR e a EMP não possuem o valor zero como opção de resposta, os modelos de regressão foram fixados pela origem dos dados, sem a utilização da constante.

\section{Considerações éticas}

Este estudo foi aprovado pela Comissão de Ética em Pesquisa com Seres Humanos da UFSJ, sob processo no 8-a/2010/ CEPES. A pesquisa também foi aprovada pelas direções das instituições de saúde mental participantes. Foram assegurados aos participantes as informações referentes ao estudo, o sigilo e o anonimato dos resultados. Os sujeitos assinaram um Termo de Consentimento Livre e Esclarecido comprovando seu acordo em participar da pesquisa e receberam uma cópia dele com um número de telefone para contato com os pesquisadores.

\section{RESULTADOS}

\section{Descrição da amostra}

Foram contatados 143 pacientes para participarem da pesquisa, mas 33 deles foram excluídos por estarem em crise, apresentarem comorbidades de transtornos por déficits cognitivos ou por não terem compreendido as questões dos instrumentos utilizados. Não houve recusas dos pacientes em participar do estudo.

Os pacientes da amostra, em sua maioria, eram do sexo feminino (68,20\%), solteiros $(38,20 \%)$ e alfabetizados (97,30\%), com escolaridade predominante de ensino fundamental incompleto $(65,60 \%)$. A idade média era de 42,11 anos $( \pm 10,77)$, sendo a mínima de 19 e a máxima de 67 anos. Grande parte possuía renda própria $(59,10 \%)$, cujo valor era, na maioria dos casos $(78,50 \%)$, de um a dois salários-mínimos e as principais fontes de renda eram o auxílio governo $(21,60 \%)$ e a aposentadoria por invalidez $(21,60 \%)$.

Quarenta por cento dos pacientes tinham um diagnóstico da categoria Esquizofrenia, Transtornos Esquizotípicos e Transtornos Delirantes, conforme os critérios da CID-1038. A maioria dos pacientes $(86,40 \%)$ não apresentava comorbidades e não tinha doenças físicas $(58,20 \%)$. A idade de início do transtorno psiquiátrico foi, em média, de 28,48 anos $( \pm 12,09)$ e a duração média do transtorno psiquiátrico, de 13,66 anos ( \pm 10,83), variando entre 1 e 46 anos. A duração média do tratamento psiquiátrico era de 11,05 anos $( \pm 9,69)$, com tempo mínimo de 1 e máximo de 46 anos. Quanto ao número de crises no último ano, $53,60 \%$ dos pacientes não tinham sofrido nenhuma crise. Dos entrevistados, 50,00\% relataram que haviam sido internados em hospitais psiquiátricos, em média, 3,96 vezes no decorrer da vida $( \pm 13,88)$, variando de 1 a 30 internações. A medicação era apenas do tipo oral para a maioria dos pacientes (75,50\%), com número médio de 3,26 tipos de medicamentos $( \pm 1,31)$.

\section{Satisfação dos pacientes com os serviços}

A tabela 1 apresenta os resultados descritivos dos escores de satisfação dos pacientes para cada item da escala SATIS-BR, em termos das médias, desvios-padrão e porcentagens de pacientes insatisfeitos, mais ou menos satisfeitos e satisfeitos. Nessa tabela, também estão assinalados os itens cujos escores médios foram significativamente mais elevados, comparativamente a um ou mais itens da escala. 
Tabela 1. Escores médios e porcentagens de satisfação por itens da escala SATIS-BR e indicação das médias mais elevadas do que uma ou mais médias $(*)$

\begin{tabular}{|c|c|c|c|c|}
\hline & & INSATISFEITOS & $\begin{array}{l}\text { MAIS OU MENOS } \\
\text { SATISFEITOS }\end{array}$ & SATISFEITOS \\
\hline Itens & Média/DP & $\begin{array}{c}\text { Respostas } \\
1 \mathrm{e} 2\end{array}$ & $\begin{array}{c}\text { Resposta } \\
3 \\
\end{array}$ & $\begin{array}{c}\text { Respostas } \\
4 \text { e } 5\end{array}$ \\
\hline 1. Ter sido tratado com respeito e dignidade & $4,78(0,61)^{*}$ & $2(1,80 \%)$ & $2(1,80 \%)$ & $106(96,40 \%)$ \\
\hline 2. Ter sido ouvido com atenção pela pessoa que 0 admitiu & $4,44(0,72)$ & $1(0,90 \%)$ & $12(10,90 \%)$ & $97(88,20 \%)$ \\
\hline 3. Profissional que admitiu o paciente compreendeu o seu problema & $4,33(0,84)$ & $3(2,70 \%)$ & $11(10,00 \%)$ & $96(87,30 \%)$ \\
\hline 4. Equipe compreendeu o tipo de ajuda de que o paciente necessitava & $4,46(0,68)$ & $2(1,80 \%)$ & $6(5,40 \%)$ & $102(92,80 \%)$ \\
\hline 5. Tipo de ajuda dada pelo serviço & $4,71(0,55)^{*}$ & $1(0,90 \%)$ & $2(1,80 \%)$ & $107(97,30 \%)$ \\
\hline 6. Obtenção de informações da equipe & $4,48(0,99)$ & $5(6,50 \%)$ & $4(5,20 \%)$ & $68(88,30 \%)$ \\
\hline 7. Discussão feita sobre seu tratamento & $4,29(0,75)$ & $2(1,80 \%)$ & $10(9,10 \%)$ & $98(89,10 \%)$ \\
\hline 8. A equipe de profissionais estava ajudando & $4,72(0,56)^{*}$ & - & $6(5,40 \%)$ & $104(94,60 \%)$ \\
\hline 9. Considera equipe amistosa & $4,58(0,61)^{*}$ & $1(0,90 \%)$ & $4(3,70 \%)$ & $105(95,40 \%)$ \\
\hline 10. Competência da equipe & $4,55(0,62)^{*}$ & - & $7(6,40 \%)$ & $103(93,60 \%)$ \\
\hline 11. Competência do terapeuta principal & $4,50(0,71)$ & $2(1,80 \%)$ & $8(7,30 \%)$ & $100(90,90 \%)$ \\
\hline 12. Conforto e aparência do serviço & $4,25(0,85)$ & $6(5,50 \%)$ & $8(7,30 \%)$ & $96(87,20 \%)$ \\
\hline 13. Condições gerais das instalações do serviço & $4,19(0,80)$ & $2(1,80 \%)$ & $17(15,50 \%)$ & $91(82,70 \%)$ \\
\hline
\end{tabular}

$F=7,44 ; p<0,01$.

As análises dos resultados de porcentagens, por itens, mostraram que a maioria dos dados se concentrou nos escores 4 e 5, que indicam satisfação e muita satisfação com o serviço, respectivamente. A maioria dos pacientes $(82,70 \%$ a 97,30\%) relatou estar satisfeita ou muito satisfeita em relação aos diversos aspectos avaliados. Para o item 6 , referente à obtenção de informações da equipe, têm-se dados de porcentagem apenas para 77 dos pacientes, já que os outros 33 sujeitos relataram nunca terem pedido informações ao serviço.

A satisfação dos pacientes foi significativamente mais elevada em relação à maneira como eles foram tratados, em termos de respeito e dignidade (item 1), comparativamente aos itens $2,3,7,12$ e 13 da escala SATIS-BR $(p \leq 0,02)$. Os itens que avaliaram a satisfação dos pacientes com a ajuda fornecida pela equipe (item 8) e com o tipo de ajuda dada pelo serviço (item 5) obtiveram médias mais elevadas do que os itens $3,7,12$ e 13 ( $p \leq 0,02)$. O escore médio de satisfação dos pacientes também foi significativamente mais elevado para o item 9, que avaliava se a equipe era amigável, em comparação com os itens 12 e $13(p \leq 0,04)$. A satisfação dos pacientes com a competência da equipe (item 10) também foi significativamente mais elevada do que em relação às condições gerais das instalações, avaliadas pelo item $13(p=0,01)$.

\section{Percepção de mudança em função do tratamento}

A tabela 2 apresenta os dados obtidos para cada item da escala EMP, em termos dos escores médios de percepção de mudança, desvios-padrão e das porcentagens dos sujeitos que apresentaram melhora, piora e ausência de mudança. Nessa tabela, também estão destacados os escores médios dos itens de percepção de mudança que foram significativamente mais elevados do que um ou mais itens da escala.

Com relação aos resultados das porcentagens, pode-se observar que a maioria dos dados se situou nas categorias

Tabela 2. Escores médios e porcentagens de percepção de mudança por itens da escala EMP e indicação das médias mais elevadas do que uma ou mais médias $(*)$

\begin{tabular}{|c|c|c|c|c|}
\hline Itens & Média (desvio-padrão) & Pior do que antes (\%) & Sem mudança $(\%)$ & Melhor do que antes $(\%)$ \\
\hline 1. Problemas pessoais & $2,77(0,50)^{*}$ & 4,50 & 30,90 & 64,60 \\
\hline 2. Humor & $2,78(0,44)^{*}$ & 3,60 & 15,50 & 80,90 \\
\hline 3. Estabilidade das emoções & $2,60(0,61)^{*}$ & 6,30 & 27,30 & 66,40 \\
\hline 4. Confiança em si mesmo & $2,65(0,58)^{*}$ & 5,50 & 24,50 & 70,00 \\
\hline 5. Interesse pela vida & $2,71(0,54)^{*}$ & 4,50 & 20,00 & 75,50 \\
\hline 6. Capacidade de suportar situações difíceis & $2,60(0,58)^{*}$ & 0,90 & 20,00 & 79,10 \\
\hline 7. Apetite & $2,35(0,72)$ & 14,50 & 36,40 & 49,10 \\
\hline 8. Energia & $2,44(0,76)$ & 16,40 & 23,60 & 60,00 \\
\hline 9. Sono & $2,63(0,68)^{*}$ & 10,90 & 15,50 & 73,60 \\
\hline 10. Saúde física & $2,25(0,67)$ & 12,70 & 50,00 & 37,30 \\
\hline 11. Sexualidade & $2,09(0,72)$ & 21,80 & 47,30 & 30,90 \\
\hline 12. Convivência com a família & $2,55(0,66)^{*}$ & 9,10 & 26,40 & 64,50 \\
\hline 13. Convivência com amigos & $2,44(0,58)$ & 4,50 & 47,30 & 48,20 \\
\hline 14. Convivência com as outras pessoas & $2,52(0,55)$ & 2,70 & 42,70 & 54,60 \\
\hline 15. Interesse em se ocupar & $2,60(0,62)^{*}$ & 7,30 & 25,40 & 67,30 \\
\hline 16. Atividades de lazer & $2,49(0,66)$ & 9,10 & 32,70 & 58,20 \\
\hline 17. Tarefas de casa & $2,51(0,66)$ & 9,10 & 30,90 & 60,00 \\
\hline 18. Capacidade de cumprir obrigações e tomar decisões & $2,49(0,66)$ & 9,10 & 32,70 & 58,20 \\
\hline 19. Item de avaliação global & $2,94(0,25)$ & - & 6,40 & 93,60 \\
\hline
\end{tabular}

$\mathrm{F}=8,56 ; p<0,01$. 
"melhor do que antes" e "sem mudança". No que se refere à piora percebida pelos pacientes em função do tratamento, destacaram-se os itens sexualidade $(21,80 \%)$, energia ou disposição para fazer as coisas $(16,40 \%)$ e apetite $(14,50 \%)$.

As médias de percepção de mudança foram significativamente mais elevadas para o "humor" (item 2) e "problemas pessoais" (item 1), comparativamente às médias dos itens 7, 8 , 10,11 e $13(p \leq 0,01)$. As percepções de mudança em relação ao "interesse pela vida" (item 5) e ao "sentimento de confiança em si mesmo" (item 4) também foram significativamente mais elevadas do que para os itens 7,10 e $11(p \leq 0,04)$. As médias referentes ao "sono" (item 9), "estabilidade das emoções" (item 3), "capacidade de suportar situações difíceis" (item 6), "interesse em trabalhar e se ocupar com alguma coisa" (item 15) e "convivência com a família" (item 12) foram significativamente mais elevadas do que as médias dos itens 10 e $11(p \leq 0,03)$.

\section{Preditores da satisfação dos pacientes com os serviços: análise multivariada}

Serão apresentados a seguir os resultados referentes às duas análises de regressão múltipla, descritas anteriormente.

Variáveis sociodemográficas e clínicas e percepção de mudança: $\mathrm{Na}$ tabela 3, estão dispostos os resultados obtidos para a análise dos fatores associados à satisfação dos pacientes com os serviços, incluindo o escore global de percepção de mudança e todas as variáveis sociodemográficas e clínicas pré-selecionadas. Os resultados indicaram que a percepção de mudança foi a variável preditora mais importante da satisfação dos pacientes com os serviços, comparativamente às demais variáveis. Em segundo lugar, destacou-se a contribuição da variável idade dos pacientes. Essas duas variáveis explicaram 99,00\% da influência do conjunto de variáveis investigadas ao grau de satisfação ( $F=1913,03 ; p$ $<0,001)$. A satisfação dos pacientes com os serviços era, portanto, maior quando eles apresentavam maior grau de percepção de melhora global e quando eles tinham maior idade.

Dimensões da percepção de mudança: Tendo-se constatado que a percepção de mudanças foi a principal preditora da satisfação dos pacientes com os serviços, foi feita uma segunda análise de regressão, visando identificar quais, dentre as três dimensões da EMP, seriam mais preditoras da satisfação. A tabela 4 apresenta os resultados dessa análise. Duas dimensões da EMP explicaram 99,00\% da contribuição desse conjunto de variáveis ao grau de satisfação dos pacientes $(F=3855,49 ; p<0,001)$. Pacientes que perceberam mais

Tabela 3. Análise de regressão linear múltipla das variáveis associadas à satisfação dos pacientes com os serviços de saúde mental

\begin{tabular}{lccccc}
\hline \multicolumn{1}{c}{ Variáveis preditoras } & Beta & Erro-padrão & $\mathbf{t}$ & $p$ & \\
\hline 1. EMP Global & 0,71 & 0,09 & 13,57 & 0,00 & $\mathrm{R}^{2}=0,99$ \\
2. Idade & 0,29 & 0,01 & 5,49 & 0,00 & $\mathrm{~F}(1913,03)$ \\
& & & & & $=510,19$ \\
& & & & & $(p=0,00)$ \\
\hline
\end{tabular}

Tabela 4. Análise de regressão linear múltipla das subescalas de percepção de mudança (EMP) associadas à maior satisfação dos pacientes

\begin{tabular}{|c|c|c|c|c|c|}
\hline Variáveis preditoras & Beta & $\begin{array}{l}\text { Erro- } \\
\text { padrão }\end{array}$ & $t$ & $p$ & \\
\hline $\begin{array}{l}\text { 1. EMP - Relacionamentose } \\
\text { Estabilidade Emocional }\end{array}$ & 0,50 & 0,14 & 6,25 & 0,00 & $\begin{array}{l}R^{2}=0,99 \\
F(3855,49)\end{array}$ \\
\hline $\begin{array}{l}\text { 2. EMP - Aspectos Psicológicos } \\
\text { e Sono }\end{array}$ & 0,50 & 0,13 & 6,22 & 0,00 & $\begin{array}{l}=1101,76 \\
(p=0,00)\end{array}$ \\
\hline
\end{tabular}

mudanças nas subescalas "Relacionamentos e Estabilidade Emocional" e "Aspectos Psicológicos e Sono" estavam mais satisfeitos com os serviços de saúde mental.

\section{DISCUSSÃO}

O presente estudo possibilitou identificar os fatores associados à satisfação dos pacientes com os serviços de saúde mental. O principal resultado obtido é que a satisfação dos pacientes foi determinada, principalmente, pelo grau de mudança percebida em função do tratamento. As pesquisas internacionais encontradas, que investigaram a relação entre a percepção de mudanças pelos pacientes e sua satisfação com os serviços de saúde mental, tiveram resultados semelhantes, ao confirmar relações significativas entre essas duas variáveis ${ }^{7,16,19-21}$. A consonância entre os resultados do presente estudo e os achados da literatura internacional reforça a teoria de que a percepção de resultados positivos do tratamento é uma forte preditora da satisfação dos pacientes com os serviços de saúde mental.

Com relação aos demais fatores associados à satisfação, a idade do paciente foi a segunda variável preditora mais importante. Pacientes mais velhos apresentaram maior grau de satisfação com os serviços do que pacientes mais jovens. Resultados semelhantes foram encontrados em pesquisas internacionais. Rosenheck et al..$^{39}$ também encontraram, em seu estudo, que, dentre as características sociodemográficas dos pacientes, a maior idade foi o fator associado mais importante da satisfação com o serviço de saúde mental avaliado. Em uma metanálise, Hall e Dornan ${ }^{40}$ encontraram relações consistentes e significativas entre a maior idade do paciente e a sua satisfação com serviços médicos. No Brasil, Souza ${ }^{41}$ confirmou, em seu estudo, que a maior idade do paciente foi um fator preditor importante da satisfação com os serviços de saúde. A consonância dos resultados dos vários estudos indica que a maior idade é um preditor importante da satisfação dos pacientes com os serviços de saúde e de saúde mental. Uma hipótese para explicar essa relação é a de que as pessoas mais idosas podem ter menores expectativas com os serviços e, consequentemente, se declararem mais satisfeitas com eles, tendo em vista que a expectativa constitui um dos fatores que determinam o grau de satisfação dos pacientes ${ }^{42}$. 
Os resultados do presente estudo indicaram um alto grau de satisfação dos pacientes com os serviços de saúde mental. Esses dados estão de acordo com os resultados da literatura internacional revisados por Weiss ${ }^{43}$ e Ruggeri ${ }^{9} \mathrm{e}$ também corroboraram os dados de outros estudos brasileiros que avaliaram a satisfação dos pacientes ${ }^{23-27}$. De acordo com Mercier e Corten ${ }^{42}$, ao avaliar a satisfação com o serviço, o paciente faz um julgamento sobre o seu tratamento, referindo-se aos seus valores, suas aspirações, sua situação atual e sua experiência com outros serviços. No presente estudo, os fatores que contribuíram para a alta satisfação, segundo relatos dos pacientes, nas entrevistas, foram: a disponibilidade de serviços gratuitos, a continuidade dos cuidados e a isenção de custos com os medicamentos. Esperidião e $\operatorname{Trad}^{44}$, em uma revisão de estudos, apontaram que a disponibilidade de medicamentos e vacinas é uma justificativa da satisfação dos pacientes com os serviços de saúde.

No presente estudo, constatou-se que os pacientes estavam significativamente mais satisfeitos com alguns aspectos dos serviços, tais como: serem tratados com respeito e dignidade, perceberem que a equipe o estava ajudando frequentemente e considerarem que obtiveram o grau de ajuda necessário no serviço. Esses resultados corroboraram os dados dos estudos de Heckert et al. ${ }^{24}$, de Kantorski et al..$^{25} \mathrm{e}$ de Camilo et al. ${ }^{27}$, que encontraram níveis elevados de satisfação para esses mesmos itens. Por outro lado, observou-se menor satisfação com as condições gerais das instalações do serviço, conforto e aparência do serviço e com o nível de discussão dos profissionais a respeito do tratamento, corroborando os dados obtidos pelo estudo de Kantorski et al. ${ }^{25}$.

Com relação à percepção de mudança pelos próprios pacientes, encontrou-se que a melhora percebida foi significativamente mais elevada em relação ao humor, problemas pessoais, interesse pela vida, sentimento de confiança em si mesmo, qualidade do sono, estabilidade das emoções e capacidade de suportar situações difíceis. Resultados muito semelhantes foram encontrados por estudos brasileiros realizados anteriormente por Cesari e Bandeira ${ }^{17}$ e Costa et al. ${ }^{18}$. O item "problemas pessoais" foi associado, pelo paciente, à sintomatologia do transtorno psiquiátrico, tanto nas entrevistas deste estudo quanto no estudo precedente que envolveu a percepção de mudanças ${ }^{18}$.

Quanto às percepções de piora encontradas no presente estudo, dois itens se destacaram: a sexualidade e a saúde física dos pacientes. Esses resultados foram encontrados, também, nos estudos brasileiros de Cesari e Bandeira ${ }^{17}$ e de Costa et al..$^{18}$ e no contexto internacional por Perreault et al. ${ }^{7}$. De acordo com Cordás e Laranjeiras ${ }^{45}$, essa piora na sexualidade e em outros aspectos físicos como energia, saúde física e apetite pode estar associada aos efeitos colaterais dos psicofármacos. Esses efeitos colaterais são fatores que ajudam a explicar a não adesão dos pacientes psiquiátricos ao tratamento ${ }^{46}$. Torna-se importante, portanto, o monitoramento dos efeitos colaterais dos psicofármacos pelos profissionais de saúde mental e por clínicos, tendo em vista que eles constituem um dos principais fatores de abandono do tratamento e, consequentemente, de recaída dos pacientes ${ }^{45}$.

Este estudo possui algumas limitações em relação à possibilidade de generalização dos resultados, uma vez que a amostra estudada não foi selecionada aleatoriamente da população-alvo. Além disso, por ser um estudo correlacional, é necessário ter cautela na interpretação dos resultados, já que esse tipo de estudo não permite o estabelecimento de relações causais entre as variáveis estudadas. Outra limitação é que, pelo fato de o presente estudo ser do tipo transversal, não é possível acompanhar a evolução da percepção de mudança e da satisfação em momentos distintos do tempo ao longo do tratamento. Pesquisas longitudinais com essa mesma temática poderão fornecer informações adicionais sobre a relação entre as variáveis. Contudo, apesar das limitações, o presente estudo destacou a importância da percepção de resultados pelos próprios pacientes e o impacto dessa variável como fator preditor da sua satisfação com os serviços de saúde mental.

\section{CONCLUSÃO}

Os resultados deste estudo indicaram a presença de variáveis sociodemográficas e clínicas importantes na predição da satisfação dos pacientes com os serviços de saúde mental. Destacou-se que a medida de percepção de mudanças pelos próprios pacientes é a principal preditora de sua satisfação com os serviços. Esse resultado indica que a medida de percepção de mudanças é relevante para apontar aspectos do tratamento que necessitam ser melhorados, visando aumentar o nível de satisfação dos pacientes. Essa avaliação é importante, tendo em vista que a satisfação está relacionada com a utilização dos serviços e com a adesão ao tratamento.

Este estudo identificou, ainda, os aspectos da vida dos pacientes em que eles perceberam melhora, ausência de mudança e piora, como resultado do tratamento recebido. A maior percepção de piora ocorreu em relação aos itens sexualidade, saúde física e apetite, possivelmente por causa dos efeitos colaterais dos medicamentos. Uma maior atenção e o controle dessas variáveis pelos profissionais dos serviços são importantes, uma vez que elas podem estar relacionadas com a menor satisfação dos pacientes, o que prejudicaria sua adesão ao tratamento. Estudos futuros poderão esclarecer a relação entre satisfação, percepção de mudança e adesão dos pacientes ao tratamento psiquiátrico.

Este estudo comprovou ser possível utilizar as medidas de resultado relatadas por pacientes psiquiátricos estabilizados. Essas medidas podem ser úteis para melhorar a qualidade dos serviços prestados. Portanto, a perspectiva dos pacientes deve ser considerada tanto em estudos de avaliação quanto na rotina dos serviços. Estudos futuros, que utilizem essas e outras medidas de resultado, na perspectiva dos próprios pacientes, podem ajudar a reunir informações úteis para a prática dos serviços, conforme recomenda a Organização Mundial de Saúde³. 


\section{CONFLITO DE INTERESSES}

Nenhum.

\section{FINANCIAMENTO}

Reestruturação e Expansão das Universidades Federais (Reuni) e Fundação de Amparo à Pesquisa do Estado de Minas Gerais (Fapemig).

\section{REFERÊNCIAS}

1. Gonçalves S, Fagundes P, Lovisi G, Lima LA. Avaliação das limitações no comportamento social em pacientes psiquiátricos de longa permanência. Ciência e Saúde Coletiva. 2001;6(1):105-13.

2. Donabedian A. Evaluating the quality of medical care. Milbank Memorial Fund Quarterly. 1966;44(3):166-203

3. Organização Mundial de Saúde. Relatório Mundial da Saúde. Saúde mental: nova concepção, nova esperança. Lisboa: Divisão de Saúde Mental da OMS; 2001.

4. World Health Organization. The World Health Report - 2008: Primary Health care now more than ever. Geneva: Division of Mental Health WHO; 2008.

5. Hunter R, Cameron R. The Scottish Schizophrenia Outcomes Study - A new paradigm for utilizing self-report assessments from patients with schizophrenia. Psychiatry in Europe. 2008:1(2):7-9.

6. Mercier L, Landry M, Corbière M, Perreault M. Measuring clients perception as outcome measurement. In: Roberts AR, Yeager KR, editors. Evidence-based Practice Manual: Research an outcome Measures in Health and Human Services. New York: Oxford University Press; 2004. p. 904-9.

7. Perreault M, White ND, Fabrès E, Landry M, Anestin AS, Rabouin D. Relationship between perceived improvement and treatment satisfaction among clients of a methadone maintenance program. Evaluation and Program Planning. 2010;33(4):410-7.

8. McCabe R, Saidi M, Priebe S. Patient-reported outcomes in schizophrenia. Br J Psychiatry. 2007;191:21-8

9. Ruggeri M. Patients' and relatives' satisfaction with psychiatric services: the state of the art of its measurement. Social Psychiatry and Psychiatric Epidemiology. 1994;29:212-27.

10. Ruggeri M. Satisfaction with mental health services. In: Thornicroft C, Tansella M, editors. Mental Health Outcome measures. London: RCPsych Publications; 2010. p. 99-115.

11. Perreault M, Katerelos TE, Sabourin E, Leichner P, Desmarais J. Information as a distinct dimension for satisfaction assessment of outpatient psychiatric services. Int J Health Care Qual Assur Inc Leadersh Health Serv. 2001;14(3):111-20.

12. Anderson R, Barbara A, Feldman $S$. What patients want: a content analysis of key qualities that influence patient satisfaction. J Med Pract Manage. 2007;22:255-61.

13. Eisen S, Ranganathan G, Seal P, Spiro A. Measuring clinically meaningful change following mental health treatment. J Behav Health Serv Res. 2007;34(3):272-89.

14. Andrade MCR, Bandeira M, Perreault M, Angelico AP, Oliveira MS. The Relationship Between Symptomatic Changes and Perceived Improvement Among Patients Treated in Brazilian Community Mental Health Services. Psychiatr Q. 2012 Jan 25.

15. Lunnen KM, Ogles BM. A multiperspective, multivariable evaluation of reliable change. J Consult Clin Psychol. 1998;66(2):400-10

16. Lunnen KM, Ogles BM, Pappas LN. A multiperspective comparison of satisfaction, symptomatic change, perceived change, and end-point functioning. Professional Psychology: Research and Practice. 2008;29(2):145-52.

17. Cesari L, Bandeira M. Avaliação da qualidade de vida e percepção de mudança em pacientes com esquizofrenia. J Bras Psiquiatr. 2010;59(4):293-301.

18. Costa CS, Bandeira M, Cavalcanti RLA, Scalon JD. A percepção de pacientes e familiares sobre os resultados do tratamento em serviços de saúde mental. Cad Saude Publica. 2011;27(5):995-1007.

19. Holcomb WR, Parker JC, Leong G, Thiele J, Higdon J. Customer satisfaction and self-reported treatment outcomes among psychiatric inpatients. Psychiatr Serv. 1998:49(7):929-34
20. Hasler G, Moergeli H, Bachmann R, Lambreva E, Buddeberg C, Schnyder U. Patient satisfaction with outpatient psychiatric treatment: the role of diagnosis, pharmacotherapy, and perceived therapeutic change. Can J Psychiatry. 2004;49(5):315-21.

21. Hasler G, Moergeli H, Schnyder U. Outcome of psychiatric treatment: what is relevant for our patients? Compr Psychiatry. 2004;45(3):199-205.

22. Perreault M, Pawliuk N, Velleux R, Rousseau M. Qualitative assessment of mental health service satisfaction: strengths and limitations of a self-administered procedure. Community Ment Health J. 2006;42(3):233-42.

23. Jaegger RC, Guitton AP, Lyrio JM, Santos MM, Freitas RCO, Goncalves SR, et al. A experiência de morar fora: avaliação da satisfação de usuários em um serviço de saúde mental. Cad Saúde Coletiva. 2004;12(1):27-39.

24. Heckert U, Teixeira LS, Trindade AS. Avaliação da satisfação dos usuários do Centro Regional de Referência em Saúde Mental (CRRESAM) da região central de Juiz de Fora, MG. HU Revista. 2006;32(1):15-9.

25. Kantorski LP, Jardim VR, Wetzel C, Olschowsky A, Schneider JF, Heck RM, et al. Satisfação dos usuários dos centros de atenção psicossocial da região Sul do Brasil. Rev Saude Publica. 2009;43(Supl 1):29-35.

26. Libério MMA. Estudo da satisfação com os CAPS da cidade do Rio de Janeiro: ouvindo seus atores principais [dissertação]. Rio de Janeiro (RJ): Universidade Federal do Rio de Janeiro; 1999.

27. Camilo CA, Bandeira M, Leal RMAC, Scalon JD. Avaliação da satisfação e sobrecarga em um serviço de saúde mental. Cien Saude Colet. 2012;20(1):82-92.

28. Selltiz C, Wrightsman L, Cook S. Métodos de pesquisa nas relações sociais. São Paulo: EPU; 1987.

29. Contandriopoulos AP, Champagne F, Potvin L, Denis JL, Bouyle P. Saber preparar uma pesquisa. São Paulo: Hucitec Abrasco; 1994.

30. World Health Organization. WHO-SATIS consumer's and caregivers' satisfaction with mental health services a multisite study. Geneva: Mental Health World Organization; 1996

31. Bandeira M, Pitta AMF, Mercier C. Escala de avaliaçãa da satisfação dos usuários em serviços de saúde mental: Satis-br. J Bras Psiquiatr. 2000;49(8):293-300.

32. Bandeira M, Andrade MCR, Costa CS, Silva MA. Percepção dos pacientes sobre o tratamento em serviços de saúde mental: validação da Escala de Mudança Percebida. Psicologia: Reflexão e Crítica. 2011;24(2):236-44.

33. Guillemin F, Bombardier C, Beaton D. Cross-Cultural adaptation of health-related quality of life measures: literature review and proposed guidelines. J Clin Epidemiol. 1993:46(12):1417-32.

34. Hosmer DW, Lemeshow S. Applied logistic regression. New York: John Wiley and Sons; 2000.

35. Dancey CP, Reidy J. Estatística sem matemática para Psicologia. Usando SPSS para Windows. Porto Alegre: Artmed; 2006.

36. Marques de Sá J. Applied Statistics Using SPSS, STATISTICA, MATLAB and R. New York: Springer Verlag; 2007.

37. Montgomery D, Peck EA. Introduction to linear regression analysis. New York: John Wiley and Sons; 1992.

38. Organização Mundial de Saúde. CID-10: Classificação Internacional de Doenças e problemas relacionados à saúde. São Paulo: Edusp; 1998.

39. Rosenheck R, Wilson NJ, Meterko M. Influence of patient and hospital factors on consumer satisfaction with inpatient mental health treatment. Psychiatr Serv. 1997;48(12):1553-61.

40. Hall JA, Dornan MC. Patient sociodemographic characteristics as predictors of satisfaction with medical care: a meta-analysis. Soc Sci Med. 1990;30(7):811-8.

41. Souza EM. A satisfação dos idosos com os serviços de saúde: um estudo de prevalência e de fatores associados em Taguatinga, Distrito Federal [dissertação]. Universidade de Brasília; 1997.

42. Mercier C, Corten P. Évaluation de la qualité de vie de patients psychotiques. In: Kovess V, editor. Evaluation de la qualité en psychiatrie. Paris: Economica; 1994. p. 263-87.

43. Weiss GL. Patient satisfaction with primary medical care: evaluation of sociodemographic and predispositional factor. Med Care. 1988;26:383-92.

44. Esperidião M, Trad LAB. Avaliação de satisfação de usuários: considerações teórico-conceituais. Cad Saude Publica. 2006;22(6):1267-76.

45. Cordás TA, Laranjeiras M. Efeitos colaterais dos psicofármacos na esfera sexual. Rev Psiq Clín. 2006:33(3):168-73

46. Santin A, Ceresér K, Rosa A. Adesão ao tratamento no transtorno bipolar. Rev Psiq Clín. 2005:32(Supl 1):105-9. 